\title{
Artefact
}

Techniques, histoire et sciences humaines

2| 2014

Art et industrie : les enjeux de la formation (XVIII $\mathrm{XX}$ ' siècles) / Qu'est-ce qu'un outil simple?

\section{Isabelle Laboulais, La Maison des mines : genèse révolutionnaire d'un corps d'ingénieurs civils (1794-1814)}

Rennes, Presses universitaires de Rennes, Collection Carnot, 2012

\section{Marie-Noëlle Maisonneuve}

\section{(2) OpenEdition}

Journals

\section{Édition électronique}

URL : https://journals.openedition.org/artefact/8705

DOI : 10.4000/artefact.8705

ISSN : 2606-9245

\section{Éditeur :}

Association Artefact. Techniques histoire et sciences humaines, Presses universitaires du Midi

\section{Édition imprimée}

Date de publication : 11 septembre 2014

Pagination : 212-215

ISBN : 978-2-271-08150-6

ISSN : 2273-0753

\section{Référence électronique}

Marie-Noëlle Maisonneuve, «Isabelle Laboulais, La Maison des mines : genèse révolutionnaire d'un corps d'ingénieurs civils (1794-1814) », Artefact [En ligne], 2 | 2014, mis en ligne le 11 mai 2021, consulté le 24 août 2021. URL : http://journals.openedition.org/artefact/8705; DOI : https://doi.org/10.4000/ artefact.8705

Ce document a été généré automatiquement le 24 août 2021.

\section{c) () $\Theta$}

Artefact, Techniques, histoire et sciences humaines est mise à disposition selon les termes de la Licence Creative Commons Attribution - Pas d'Utilisation Commerciale - Pas de Modification 4.0 International. 


\section{Isabelle Laboulais, La Maison des mines : genèse révolutionnaire d'un corps d'ingénieurs civils (1794-1814)}

Rennes, Presses universitaires de Rennes, Collection Carnot, 2012

Marie-Noëlle Maisonneuve

\section{RÉFÉRENCE}

Isabelle Laboulais, La Maison des mines : genèse révolutionnaire d'un corps d'ingénieurs civils (1794-1814), Rennes, Presses universitaires de Rennes, Collection Carnot, 2012, 376 p.

1 En dehors des articles universitaires, les quelques ouvrages existant sur l'histoire de l'École et du Corps des mines sont le plus souvent des travaux de seconde main. D'où tout l'intérêt du livre d'Isabelle Laboulais, paru fin 2012 à la suite de sa soutenance d'HDR, dans lequel elle porte un regard attentif d'historienne sur la Maison des mines, ce curieux objet d'origine révolutionnaire, un lieu, deux lieux en fait - nous reviendrons sur cet important détail -, mais aussi une communauté en gestation, un groupe d'experts, un Corps en devenir...

2 La Maison des mines fonctionne entre l'an II et 1814. C'est en 1794 que l'Agence des mines nouvellement créée prend possession (pour sa mission ainsi définie : «Le Comité de Salut Public a vu que l'intérêt de la Liberté exigeait que les mines fussent vivifiées. Une Agence a été organisée... Les artistes qui la composent... versés dans toutes les parties de l'Art des mines... Cette Agence préside à une Maison d'instruction... »), de deux hôtels particuliers contigus, l'hôtel de Mouchy et l'hôtel de Périgord, l'un destiné à la maison d'instruction, l'autre à l'administration. Du lieu à proprement parler, il ne reste rien: les deux hôtels ont été détruits lors du percement du boulevard SaintGermain et ils ne sont représentés sur aucune gravure. De cette Maison des mines ne 
demeurent que des archives, et c'est à travers leur analyse extrêmement fine et pertinente qu'Isabelle Laboulais parvient à lui redonner vie, à reconstituer l'esprit qui y régnait, les conflits qu'elle a affrontés, les nostalgies qu'elle a laissées.

Isabelle Laboulais démontre à quel point la Maison des mines peut s'analyser à sa création comme un «lieu d'utopie », un lieu de «fédération de savoirs élaborés dans l'idéalisme de l'an II » pour constituer le cœur de «la science des mines », qui se veut différente de l'art de l'exploitation. Au contraire des écoles minières germaniques, cette structure ne se situe pas sur un lieu d'extraction, mais dans un lieu de pouvoir. Car elle est créée à une époque où le Comité de salut public est convaincu du rôle indispensable de la science et de son enseignement pour exploiter les ressources du sous-sol dont la République a fortement besoin. Deux portes de communication, symboles forts des échanges indispensables entre administration et maison d'instruction, sont immédiatement percées entre les deux anciens hôtels particuliers alors que l'École royale des mines à la Monnaie n'avait jamais été associée à l'administration des mines.

L'auteur revient alors sur cette «science des mines » revendiquée si hautement par les hommes de l'an II. Si, au cours du XVIII ${ }^{\mathrm{e}}$ siècle, les mines sont devenues progressivement un domaine où intervient l'administration de l'État, en 1791, elles sont désormais à la disposition de la Nation et le système de la concession est généralisé. En 1794, avec l'isolement forcé de la France, les ingénieurs des mines, dont la remise en cause a été très violente en 1790, trouvent leur pleine utilité aux yeux du Comité de salut public. Cependant la méfiance marquée de l'époque révolutionnaire envers le corporatisme pousse à adopter, pour faire figure de relais entre le pouvoir central et les exploitants, une structure souple tenant plutôt du groupe d'experts. Les membres de l'Agence résident à Paris les quatre mois d'hiver, mais sont tous censés voyager pendant huit mois : missions de contrôle et d'encadrement, connaissances et explorations minières. Ce groupe d'experts est décrit par Isabelle Laboulais comme assez informel, attaché également à d'autres cercles comme la Société d'histoire naturelle de Paris ou la Société philomatique. La culture commune qu'il élabore peu à peu repose sur une instance très collégiale et plutôt consensuelle: la Conférence des mines, qui met en œuvre une pratique hybride entre usages académiques - dont la liberté de parole - et procédures administratives, ne se veut lieu d'autorité auprès du pouvoir que pour juger de la recevabilité des projets d'exploitation, appuyée sur les expertises du laboratoire dirigé par Vauquelin, et non pour juger des savoirs minéralogiques qui y sont librement discutés. Si, en passant par les bureaux, les savoirs savants peuvent devenir savoir de gouvernement, seule la maîtrise de la science donne la compétence technique: la pratique ne peut la produire. Quant à la Maison d'instruction, elle apparaît à travers ses archives comme un lieu d'apprentissage inséré dans le cadre d'une sociabilité douce encourageant les rapports étroits et familiers entre disciples et professeurs-savants, dont la plupart des élèves semblent avoir gardé un souvenir ému.

L'étude constate que cette conception est rapidement mise à mal. En 1795, l'Agence des mines, devenue Conseil des mines, est réduite à des fonctions consultatives et passe sous la tutelle du ministère de l'Intérieur. L'enjeu devient de savoir si le Conseil administre les mines ou se contente de les surveiller, et des conflits d'attribution s'en suivent : administrer est en train de devenir un métier et les ingénieurs-savants n'ont pas de compétences administratives. En outre, la création de l'École centrale des travaux publics, ultérieurement École poly-technique, soulève la question de la place de l'enseignement savant à la Maison d'instruction. En 1802, le départ des élèves pour 
Pesey achève de remettre en cause cette culture d'étroite proximité entre science et administration, en attribuant uniquement la "pratique » à l'École des mines exilée en tant qu'école d'application; cela fera l'objet d'une résistance acharnée au niveau de l'enseignement, ce que démontre Isabelle Laboulais en indiquant qu'en marge des statuts, des cours abstraits sont maintenus : minéralogie, géologie, chimie, langues. Les ingénieurs des mines sont recrutés de manière désormais homogène, la résidence en province est généralisée et Chaptal s'efforce visiblement de les recentrer sur leurs fonctions pratiques. Dans cette perspective, le port imposé de l'uniforme est assez symptomatique et Isabelle Laboulais note que les savants Hauÿ et Vauquelin rejoignent le Muséum d'histoire naturelle.

6 En 1805, le Conseil rappelle, alors que le directeur général cherche à imposer son opinion, qu'il s'assimile à un jury et offre donc une garantie d'impartialité et d'équité des avis. Cette affirmation du modèle de la société savante a lieu alors même qu'on se dirige vers la fin de l'identité savante de l'institution. En 1810, Laumont, conseiller d'état et préfet de Seine-et-Oise, est choisi pour diriger le Conseil général de mines : la maitrise des savoirs administratifs l'emporte et on voit l'accroissement de la subordination des agents, la reprise du principe du stationnement et l'affirmation d'une structure très hiérarchisée du Corps. Certes, la reconnaissance - y compris celle de l'expertise judiciaire - de l'ingénieur des mines témoigne du statut acquis en vingt ans par le Corps, mais désormais cette reconnaissance tient davantage à l'autorité qui lui est dévolue qu'aux savoirs experts. Isabelle Laboulais va jusqu'à évoquer «le spectre de la maison des mines ", puis rappelle qu'en 1814 encore, un mémoire signé collectivement par les membres du Corps des mines montre qu'ils n'arrivent pas à faire «le deuil» de ce type de structure. Dans ce mémoire, ils continuent d'opposer ingénieurs et administrateurs et proposent la création d'un poste de "premier ingénieur " pour gérer la direction des études, la conservation des objets relatifs aux études, au " perfectionnement et à la science de mines »; ils tendent à reconstituer la Conférence des mines en suggérant que tout élève ou ingénieur se trouvant à Paris devrait assister aux séances du Conseil; ils cherchent aussi à imposer que tout directeur d'exploitation ait étudié trois ans dans une école pratique ou qu'il ait reçu de l'administration un certificat attestant être bien instruit. Si, en novembre 1810, la reconnaissance du rôle des ingénieurs des mines dans l'administration publique a lieu, c'est au prix du renoncement de l'identité singulière bâtie en l'an II où instruction, administration et exploitation relevaient d'une seule et même mission, comme le démontre clairement Isabelle Laboulais.

7 La Maison des mines, durant ses vingt années d'existence comme lieu de convergence de savoirs experts, joue pleinement le rôle de dépôt central au service des ingénieurs des mines par les archives qu'elle abrite, la bibliothèque et les collections qu'elle met à la disposition de ses membres, et Isabelle Laboulais s'intéresse longuement à ces aspects révélateurs de la volonté de regrouper, mais aussi de faire circuler largement la science des mines.

8 La gestion des archives de travail de la communauté s'y effectue. Comme le rapport de l'ingénieur de l'époque révolutionnaire est rarement structuré en rubriques bien identifiées, l'emprise de la description étant encore très forte, on constate, dès l'an III, la création de fiches plus synthétiques. L'intérêt pour l'élaboration et la conservation des cartes qui permettent le "coup d'œil sur le territoire " et se révèlent de remarquables outils de synthèse, est constant. Centralisant les connaissances sur les 
mines et les exploitations, le Corps se donne ainsi les moyens de les administrer et a pleine conscience de l'importance de ses archives comme outil de travail et de pouvoir.

Dans la même perspective, Isabelle Laboulais souligne les difficultés de la création d'une bibliothèque spécialisée, réellement axée sur la science des mines par prélèvement dans des dépôts littéraires trop généralistes : les bibliothécaires successifs tentent d'obtenir les collections vraiment spécialisées qui conviendraient, comme celles de Lavoisier et de Dietrich.

10 Le classement des collections minéralogiques, constituées elles aussi par confiscation, puis considérablement étoffées par les collectes de terrain, met en valeur, sur quatre ensembles, deux collections principales bien symptomatiques de la double mission de l'Agence des mines: une collection méthodique pour l'instruction et la science, une collection géographique pour l'inventaire. Cette dernière est étroitement liée aux pratiques des ingénieurs et doit se comprendre comme un recueil de pièces justificatives à l'appui des rapports et des propositions du Corps des mines : au cœur même des collections, se mêlent pratiques et usages savants. Collections, archives et laboratoire sont considérés comme tellement indispensables qu'ils ne suivront pas l'École à Pesey.

11 Pour diffuser sa culture de la science des mines, et aussi revendiquer son expertise, le Conseil se dote du Journal des mines pour lequel, constate Isabelle Laboulais, d'une manière ou d'une autre, tout le personnel de l'Agence travaille sous la direction de Coquebert de Montbret. En 1813, la fabrication de tables générales montre à quel point les articles de cette publication sont indispensables au Conseil. Bien que les ingénieurs des mines insistent régulièrement sur son aspect de publication savante et que quelques controverses y figurent, dès l'an X, il est clair que les ouvrages scientifiques analysés sont souvent des manuels et que les écrits de géologie pure publiés n'émanent plus d'ingénieurs des mines. Sa diffusion relativement modeste, notamment à l'étranger, explique qu'il devienne peu à peu l'indispensable bulletin de liaison du Corps, par lequel celui-ci certes se donne à voir, mais fait aussi circuler le savoir technique, y compris en province, et tente d'inciter l'industrie à prendre des initiatives. En ce sens, le Journal des mines apparaît à Isabelle Laboulais comme une sorte de «double» de la Maison des mines : il rassemble sa communauté de moins en moins informelle et passe de la contribution vraiment scientifique à la statistique descriptive.

12 Alors que la création de l'Agence des mines repose sur la conviction révolutionnaire qu'en matière d'exploitations minières, l'impulsion doit venir de l'État, ce modèle devient caduc sous le Directoire et l'Empire; il est finalement défait, mais après bien des résistances. En 1810, "les bureaux ont gagné », dit Isabelle Laboulais: pour s'imposer socialement, l'ingénieur des mines a accepté de s'affranchir de la figure de savant qui lui a servi de modèle et le Corps des mines peut devenir une puissance reconnue mais par son rôle d'interface entre l'État et l'industrie.

13 Cet ouvrage, appuyé sur le sérieux et la précision de ses références et cependant très agréable à lire, fait brillamment percevoir, à travers l'étude de la Maison des mines, les circonvolutions de cette période charnière entre le XVIII ${ }^{e}$ et le $\mathrm{XIX}^{\mathrm{e}}$ siècle où prévaut un foisonnement intellectuel désireux d'allier théorie et pratique. 


\section{AUTEURS}

\section{MARIE-NOËLLE MAISONNEUVE}

Mines ParisTech 